\title{
Radiative Microwave Heating of Hyperthermia Therapy on Breast Cancer in a Porous Medium
}

\author{
${ }^{1}$ S.I. Oke, S.O. Salawu ${ }^{2}$, M.B. Matadi ${ }^{1}$ and I.L Animasaun ${ }^{3}$ \\ ${ }^{1}$ Department of Mathematical Sciences, University of Zululand, Private Bag X1001, KwaDlangezwa, 3886, South Africa. \\ 2 Department of Mathematics, Landmark University, Omu-aran, Nigeria \\ ${ }^{3}$ Department of Mathematical Sciences, Federal University of Technology, Akure, Nigeria
}

\begin{abstract}
Cancer is a leading cause of morbidity and mortality worldwide, yet much is still unknown about its mechanism of establishment and destruction. Recently, studies had shown that tumor cells cannot survive under the high temperature conditions. This treatment technique is called Hyperthermia. This report presents the case of radiative microwave heating of hyperthermia therapy on breast cancer in a porous medium. In this study, the steady state is solved analytically while unsteady state is solved using semi-implicit finite difference to get a more accurate prediction of blood temperature distributions within the breast tissues. A moderate temperature hyperthermia treatment is apply which results into cell death due to an increase in the level of cell sensitivity to radiation therapy and blood flow in tumor and oxygen. The results show that by applying metabolic heat generation rate of $3.97 \times 10^{5} \mathrm{Wm}^{-3}$, it takes upto 2 minutes for the tumor cells to get the require therapeutic temperature point.
\end{abstract}

Keywords: hyperthermia treatment; bioheat transfer; breast cancer; radiation; biological tissue.

\section{Introduction}

Scientifically, abnormal growth of cells (i.e. malignancy) is known as cancer. Cancer is a complex life-threatening disease which affects millions of people worldwide. World Health Organization [1] remarked that the disease is a leading cause of death in developed countries and second leading cause of death in developing countries. Cancer is any malignant growth or tumor caused by abnormal and uncontrolled cell division. If not stopped, it may spread to other parts of the body through the lymphatic system or blood stream. It is caused by both internal (DNA damage, hormones imbalanced) and external (environmental such as tobacco smoking, alcoholic consumption, chemical substances etc) factors [2]. In this study, the focus shall be on female breast cancer. Breast cancer is the second most common cancer among women worldwide with nearly 1.7 million new diagnosed cases National Breast Cancer Foundation [3]. Mortality rate remains around $12.5 \%(246,660)$ among women in United State of American [4]. Breast cancer starts when cells in the breast begin to grow out of control. These cells usually form a tumor that can often be seen on an X-ray or felt as a lump. The tumor is malignant (cancerous) if the cells can grow into (invade) surrounding tissues or spread (metastasize) to distant areas of the body. Breast cancer occurs almost entirely in women, however, men can be affected as well [5]. There are three major risk factors of breast cancer in which $97 \%$ is caused by estrogen imbalance (fat cells are estrogen-making machines, leading to estrogen imbalance or dominance), $20 \%$ is caused by genetic (family history/genetic influences) and $80 \%$ is caused by environmental (poor diet, smoking tobacco, alcoholic consumption, exposure to toxins and pollutants can tip the balance) [6]

Hyperthermia is a technique for treatment of malignant and benign tumors by administering heat in different ways by the Oncologist. There are different external heat sources

\footnotetext{
${ }^{1}$ Corresponding Author, E-mail: segunoke2016@gmail.com
} 
such as radio-frequency, microwave, magnetically excitable thermo-seeds, infra-radiation and many more [7]. Habash and Coworker [9] studied temperature level and time duration of hyperthermia therapy. From fig.1, the authors grouped the hyperthermia into three categories which are (i) long term low temperature hyperthermia ( $T \leq 41^{0} \mathrm{C}$ for $6-72 \mathrm{hrs}$ ), (ii) moderate temperature hyperthermia $\left(41<T<46^{0} \mathrm{C}\right.$ for $15-60 \mathrm{mins}$ ) and (iii) high temperature hyperthermia or thermal ablation $\left(T \geq 46^{0} C\right.$ for $4-6$ mins). Thermo-therapy depend on the intensity of volumetric heat source to different kinds of hyperthermia. The site of cancerous cells determines the types of hyperthermia to be used whether localised hyperthermia, regional hyperthermia or whole body hyperthermia (WBH) in clinical therapeutic application to treat the cancerous cells [8-10]. For localised hyperthermia, high temperature is required during the treatment of small area where the cancer cells are located in the body, in order to destroy or kill the cancerous cells while in regional hyperthermia, the therapy is applied to the large area of the whole tissues or organ but in whole body hyperthermia, the therapy is applied with a special technique to treat metastatic cancerous cells when it spreads throughout the body. According to Bhownik et al. [11], heat transfer in multilayer tissues is a very complex process. It requires multiple mechanism involving conduction, convection, blood perfusion and diffusion in micro-vascular cells. The study of heat transfer and thermal damage in multilayer tissues are of great interest and can be useful in hyperthermia therapy clinical. In general, there are three fundamental bioheat transfer models such as Pennes bioheat transfer model [12], thermal wave bioheat transfer model [13],[14] and dual phase-lag bioheat transfer model available in literature [15]. The mechanism of heat decomposition and consequent increase in temperature rise in biological tissues during microwave heating [16]. Microwave heating has been found useful as a solution to many challenges facing industrial melting, sintering and drying [17]. In fact, microwaves have also been found useful in medical therapy as a means for heat deposition in hyperthermic treatment. It was discovered and reported in [18] that tumor cells are destroyed through heat deposition in tissue at a given time and at a higher temperature.

Many researchers such as:[17-20] have carried out investigation on hyperthermia therapy, in their studies, the effect of surface cooling and blood flow on the temperature distribution of tissues during microwave heating. The heating source was considered as a step function of time and thermal conductivity of the tissue to be constant. Kritikos and Coworker [20] investigated the differential temperature rise in a spherical region, stimulating a potential hot-spot in a central region of the head. Fourier transform method was used to solve the problem and the result shows that the temperature rise for a large man's head is small. It was pointed out by Hill and Pincombe [17] that some similarity temperature profiles for microwave heating of a half space and power law and exponential micro physical property (thermal conductivity, specific heat) were considered in the mathematical formulation; the differential equation was solved numerically to obtained the solution to the transient state problems. Furthermore, [21] considered the steady state microwave heating of a finite one-dimensional stability. The authors assumed that the electrical conductivity and thermal absorptivity were having Arrhenius law dependence on temperature while the electrical permittivity and permeability assumed constant. However,[22] discussed the thermal state of the biological tissue when incident with microwaves. The study considered one dimensional multilayer model and analyzed it using Maxwell equations coupled with bioheat model. Adegbile and Ogunmoyela [23] examined the effect of spatial and temperature dependent blood perfusion on the temperature field of biological tissue during microwave heating. Hence, their solutions revealed the possibility of multiple results when blood perfusion is temperature dependent, however, a unique solution was obtained when dependent temperature is on the spatial variables. Popoola and Ayeni [24], investigated the multiplicity of solutions of a boundary value problem arising from the 
theory of microwave heating of cancerous tumor. In their study, finite difference method and shooting techniques was used to prescribe an initial gradient results in the existence of a unique solution. Following the above studies, most of the authors neglected the effect of radiative heat flux and permeability of the porous media of the biological tissues. However, the present study is interested in examining the hyperthermia therapy and radiative heat flux intensity effects on the breast tissues with permeability of the porous media. The study is basically done for moderate temperature hyperthermia treatment of breast cancer in critical situation and characterized analytically to establish a more accurate prediction of blood temperature distributions within the breast tissues. Checking through the literature, it is worth remarking that the effects of variations in blood thermal conductivity, porosity, thermal radiation, heat source and blood perfusion on temperature distribution during microwave heating of hyperthermia therapy is an open question; hence this study.

\section{Mathematical formulation}

During hyperthermia therapy, the body tissue which is initially at a constant temperature $T_{0}=37^{0} \mathrm{C}$ is heated by some external heat source. Heat transfer mechanism in the living tissues involves a combination of thermal conduction in tissues, convection and perfusion of blood, and metabolic heat production. In Makinde and Animasaun [25], Radiation was considered only at the skin surface and is negligible everywhere else. Assuming local thermal equilibrium (between blood and tissue), Pennes [12] proposed a model to describe the arterial blood temperature which is uniform throughout the tissue while the venous blood temperature is equal to local tissue temperature. The one-dimensional transient energy balance equation is given as follows [22]:

$$
\rho_{b} C_{p b} \frac{\partial \bar{T}}{\partial \bar{t}}=K_{b} \frac{\partial^{2} \bar{T}^{2}}{\partial \bar{y}^{2}}+\omega_{b} \rho_{b} C_{b}\left(T_{b}-\bar{T}\right)+Q(\bar{T})|E|^{2}
$$

Additionally, the benefit of utilizing porous media theory in modeling bioheat transfer due to fewer assumptions as compared to different established bioheat transfer models are stressed by [2, 28-33].

$$
\rho_{b} C_{p b} \frac{\partial \bar{T}}{\partial \bar{t}}=K_{b} \frac{\partial^{2} \bar{T}}{\partial \bar{y}^{2}}-\frac{\partial q}{\partial \bar{y}}+\frac{\epsilon}{k}+\omega_{b} \rho_{b} C_{b}\left(T_{b}-\bar{T}\right)+Q_{m}\left(\bar{T}-T_{0}\right)
$$

Subject to the following boundary conditions:

$$
\bar{T}(\bar{y}, 0)=37^{0} C, \quad \bar{T}(0, \bar{t})=37^{0} C, \quad \bar{T}(a, \bar{t})=45^{0} C
$$

Using Rosseland diffusion approximation for radiation [28],[29]

$$
q=\frac{-4 \sigma}{3 \delta} \frac{\partial T^{4}}{\partial \bar{y}}
$$

where $\sigma$ is the Stefan-Boltzmann constant and $\delta$ is the mean absorption coefficient. It is assumed that the temperature differences within the blood flow are sufficiently small such that $T^{4}$ may be expressed as a linear function of temperature, using Taylor series to expand $T^{4}$ about the free stream $T_{\infty}$ and neglecting higher order terms, this gives the approximation

$$
T^{4} \cong 4 T_{b}^{3} \bar{T}-3 T_{b}^{4}
$$

using equation (4) and (5) to get,

$$
\frac{\partial q}{\partial \bar{y}}=\frac{-16 \sigma T_{b}^{3}}{3 \delta} \frac{\partial^{2} \bar{T}}{\partial \bar{y}^{2}}
$$


Hence, equation (2) gives

$$
\rho_{b} C_{p b} \frac{\partial \bar{T}}{\partial \bar{t}}=K_{b}\left(1+\frac{4}{3} R\right) \frac{\partial^{2} \bar{T}}{\partial \bar{y}^{2}}+\frac{\epsilon}{k}+\omega_{b} \rho_{b} C_{b}\left(T_{b}-\bar{T}\right)+Q_{m}\left(\bar{T}-T_{0}\right)
$$

The following dimensionless parameters are introduced

$$
y=\frac{\bar{y}}{a}, \quad t=\frac{\bar{t}}{a^{2}}, \quad \theta=\frac{\left(\bar{T}-T_{0}\right)}{\left(T_{b}-T_{0}\right)}
$$

Substitute the dimensionless quantities (8) in equations (3) and (7) to get

$$
\frac{\partial \theta}{\partial t}=\alpha\left(1+\frac{4}{3} R\right) \frac{\partial^{2} \theta}{\partial y^{2}}-(\gamma-\lambda) \theta+(\beta+\gamma)
$$

The corresponding boundary conditions becomes

$$
\theta(y, 0)=37^{0} C, \quad \theta(0, t)=37^{0} C, \quad \theta(1, t)=45^{0} C
$$

where $\alpha=\frac{K_{b}}{\rho_{b} C_{p b}}, \beta=\frac{a^{2} \epsilon}{\rho_{b} C_{p b}\left(T_{b}-T_{0}\right)}, \gamma=a^{2} \omega_{b}, \lambda=\frac{a^{2} Q_{m}}{\rho_{b} C_{p b}}$, and $R=\frac{4 \sigma T_{b}^{3}}{\delta K_{b}}$ are the dimensionless parameters

\section{Analytical Solution}

When $\frac{\partial \theta}{\partial t}=0$ in equation (9), the steady state of the problem is obtained. The resulting differential equation along with the boundary conditions are then solved analytically and obtained as;

$$
\begin{aligned}
& \theta(\eta)=\frac{\mathrm{e}^{\frac{\sqrt{3} \sqrt{\gamma-\lambda} \eta}{\sqrt{\alpha} \sqrt{3+4 R}}}\left(36 \mathrm{e}^{-\frac{\sqrt{3} \sqrt{\gamma-\lambda}}{\sqrt{\alpha} \sqrt{3+4 R}}} \gamma-\mathrm{e}^{-\frac{\sqrt{3} \sqrt{\gamma-\lambda}}{\sqrt{\alpha} \sqrt{3+4 R}}} \beta-37 \mathrm{e}^{-\frac{\sqrt{3} \sqrt{\gamma-\lambda}}{\sqrt{\alpha} \sqrt{3+4 R}}} \lambda-44 \gamma+\beta+45 \lambda\right)}{\left(\mathrm{e}^{\frac{\sqrt{3} \sqrt{\gamma-\lambda}}{\sqrt{\alpha} \sqrt{3+4 R}}} \gamma-\mathrm{e}^{\frac{\sqrt{3} \sqrt{\gamma-\lambda}}{\sqrt{\alpha} \sqrt{3+4 R}}} \lambda-\mathrm{e}^{-\frac{\sqrt{3} \sqrt{\gamma-\lambda}}{\sqrt{\alpha} \sqrt{3+4 R}}} \gamma+\mathrm{e}^{-\frac{\sqrt{3} \sqrt{\gamma-\lambda}}{\sqrt{\alpha} \sqrt{3+4 R}}} \lambda\right)}+ \\
& \frac{\frac{1}{\gamma-\lambda} \mathrm{e}^{-\frac{\sqrt{3} \sqrt{\gamma-\lambda} \eta}{\sqrt{\alpha} \sqrt{3+4 R}}}\left(36 \mathrm{e}^{\frac{\sqrt{3} \sqrt{\gamma-\lambda}}{\sqrt{\alpha} \sqrt{3+4 R}}} \gamma-\beta \mathrm{e}^{\frac{\sqrt{3} \sqrt{\gamma-\lambda}}{\sqrt{\alpha} \sqrt{3+4 R}}}-37 \mathrm{e}^{\frac{\sqrt{3} \sqrt{\gamma-\lambda}}{\sqrt{\alpha} \sqrt{3+4 R}}} \lambda-44 \gamma+\beta+45 \lambda\right)}{\left(\mathrm{e}^{\frac{\sqrt{3} \sqrt{\alpha-\lambda}}{\sqrt{\alpha} \sqrt{3+4 R}}}-\mathrm{e}^{-\frac{\sqrt{3} \sqrt{\gamma-\lambda}}{\sqrt{\alpha} \sqrt{3+4 R}}}\right)}+
\end{aligned}
$$

$$
\frac{\beta+\gamma}{\gamma-\lambda}
$$

\section{Numerical of Solution}

The unsteady state solution for equations (9) and (10) are obtained using a numerical method. The computational algorithm embraced for the hyperthermia therapy on the breast cancer equation is semi-implicit finite difference scheme as in [30],[31] the scheme takes implicit terms at the intermediary time level $(N+\xi)$ where $0 \leq \xi \leq 1$. In order to make immense time steps, $\xi$ is taken to be 1 . In fact, being totally implicit, the adopted numerical algorithm offered in this work is hypothesized to be appropriate for any estimation of time steps. The equations is discretarized on a cartesian linear mesh with homogenous grid on which the finite-differences are taken. Approximating the first 
and second spatial derivatives with central differences of second-order, the equation conforming to the last and first grid points are adapted to integrate the boundary conditions. The semi-implicit scheme for the hyperthermia module takes the form:

$$
\frac{\left(\theta^{(N+1)}-\theta^{(N)}\right)}{\Delta t}=\alpha\left(1+\frac{4}{3} R\right) \theta_{y y}^{(N+\xi)}-(\gamma-\lambda) \theta^{(N)}+(\beta+\gamma)
$$

The equation for $\theta^{(N+1)}$ becomes:

$$
\begin{aligned}
& -r \theta_{j-1}^{N+1}+(1+2 r+\varphi \Delta t) \theta_{j}^{N+1}-r \theta_{j+1}^{N+1}=\alpha\left(1+\frac{4}{3} R\right)\left(\theta^{(N)}+\Delta t(1-\xi) \theta_{y y}^{(N)}\right)- \\
& \Delta t(\gamma-\lambda) \theta^{(N)}+\Delta t(\beta+\gamma)
\end{aligned}
$$

where $r=\xi \Delta t / \Delta y^{2}$ while forward difference schemes are taken for all derivatives of time. The solution method for $\theta^{(N+1)}$ decreases to the tri-diagonal inversion matrices.

The scheme (2) was tested for uniformity. When $\xi=1$ enable us to take large time steps that is second order in space but first-order precise in time. As previously assumed, the scheme satisfies any time step values. The Maple code was used to carried out the numerical analysis.

\section{Results and Discussion}

A one-dimensional multilayer transient bioheat for predicting temperatures in living biological tissue such as breast tissue during microwave heating has been investigated using analytical method of solution after a linearization technique. The computational analysis has been made by using MAPLE 18 software and results are presented in figures. The typical thermophysical properties for heat transfer in biological tissue require different shapes probe, boundary conditions and heat source due to metabolic heat generation in the tissue. The selected reference value of parameters to compute the temperature profile in biological tissue in finite domain are given below : $C_{p b}=3500 \mathrm{~J} \mathrm{Kg}^{-1} \mathrm{~K}^{-1}, \rho_{b}=1060 \mathrm{Kgm}^{-3}, T_{0}=$ $37^{0} \mathrm{C}, \omega_{b}=0.00125 \mathrm{Kgs}^{-1}, K_{b}=0.24 \mathrm{Wm}^{-1} \mathrm{~K}^{-1}$ and $\mathrm{T}_{b}=45^{0} \mathrm{C}$ [22],[32]. The transient solutions on an even finer mesh $(\Delta t=0.0001$ with $\Delta y=0.01)$ are display in Fig 2a. The Figure portray a transient increase in temperature until a steady state is reached. Fig $2 \mathrm{~b}$ represent the time of achieving steady state temperature profile for different parameters values. That is, the time of obtaining no variation in the temperature distribution, the maximum temperature is recorded at $\theta_{\max }=44.977$.

Figs $3 \mathrm{a}$ and $3 \mathrm{~b}$ show the influence of variation in values of the thermal conductivity on the temperature distributions. It is observed that an increase in the thermal conductivity value increases the temperature profiles when $\lambda=0.5$ but decreases when the value of $\lambda=2$. This is because an increase in $\alpha$ causes a rise in the tissue layers thickness when $\lambda=0.5$ and thereby increase the average temperature within the tissue layers but at $\lambda=2$ the tissue layers get thinner and heat is able to diffuse away from the heated surface.

Figs $3 \mathrm{c}$ and $3 \mathrm{~d}$ depict the response of porosity parameters $\beta$ on the temperature profiles. It is observed that the temperature profiles rises as the porosity parameter increases at $\lambda=0.5$ minimum and $\lambda=2$ maximum value occurred. The reason for this behaviour is that the porosity term provides an additional support to the blood flow mechanism through the porous tissue which causes the blood cell to move at an accelerating rate. The effect of radiation parameter $R$ on the temperature profiles is shown in Fig 4a and $4 \mathrm{~b}$. It is noticed that the figures are both minimum values, whenever there is increase in $\lambda$ the rate at which temperature increases become smaller. It is seen that as the value of $R$ increases, there is corresponding increase in the temperature profiles at $\lambda=0.5$ and $\lambda=1$ this due to an increase in the rate at which heat penetrate tumor cell as a result 
of radiation. Figs $4 \mathrm{c}$ and $4 \mathrm{~d}$ depict the variation in the temperature profile in relation to the blood perfusion parameter $\gamma$. Fig $4 \mathrm{c}$ is linear in nature especially when $\lambda=0.5$ and maximum temperature is obtained as $\gamma$ increases. However, it simply means increase in the blood perfusion rate causes an increase in the temperature of the biological tissue at $\lambda=0.5$ and $\lambda=1$ Figs $5 \mathrm{a}$ and $5 \mathrm{~b}$ show the influence of variational increase in the metabolic heat source $\lambda$ on the temperature distribution. Metabolic heat generation in the tissue is used for active transport via membrane pumps, energy requiring for chemical reaction, such as muscular work, formation of the glycogen from glucose and proteins from amino acid. Almost all metallic energy used in these processes is converted into heat within the biological tissue. It is noticed that an increase in the values of $\lambda$ increases the temperature profiles at $\alpha=2$ and $\gamma=0.004$ which results in the death of cancerous cells.

\section{Conclusion}

Radiative microwave heating presents a new way to treat cancerous tumors by hyperthermia with minimal or no damage to surrounding tissues using Pennes' bioheat model to calculate the temperature distribution because of the simplicity of this model. However, its major setback is that it cannot account for the blood flow direction flow on due to the non-directional perfusion term in its model. The porosity and permeability in this case describe the direction of blood flow in the tissue, but it needs a reasonable amount of heat past a porous medium. The different values of $\lambda$ significantly affected the temperature profile at the target area of breast cancer during treatment as well as radiative heat flux in the control and prediction of temperature at targeted region in treatment process of breast cancer cells. As the values of metabolic heat source parameters increases, temperature at target area also increases. Thus, this helps in therapy treatment process. The effect of boundary conditions on temperature profile is reflected on the thermal conductivity with high temperature at the outer surface that is being controlled by cooling pad.

Finally, rigorous mathematical analysis can help in controlling heat during hyperthermia treatment. Therefore, it is believe that hyperthermia treatment based on radiative microwave heating of bioheat transfer model is important in clinical or medical practice and Oncologist expert should embrace it for alternative treatment because it is affordable.

\section{Declarations}

\section{Author's Contribution}

The authors contributed significantly in writing this paper. All authors read and approved the final manuscript for submission.

\section{Acknowledgment}

The authors are thankful to the Institutional Research Fund of University of Zululand, South Africa.

\section{Competing Interest}

The authors declare that there is no conflict of interests regarding the publication of this paper. 


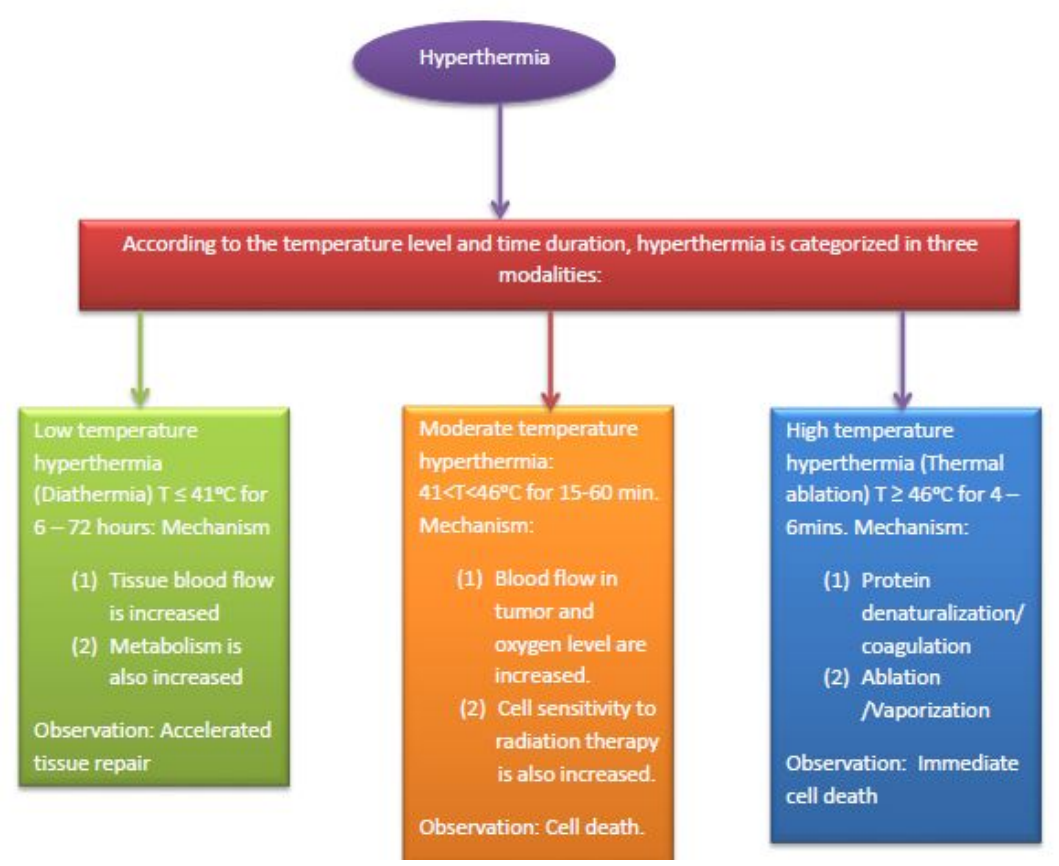

Figure 1: $\quad$ Types of the hyperthermia [9]

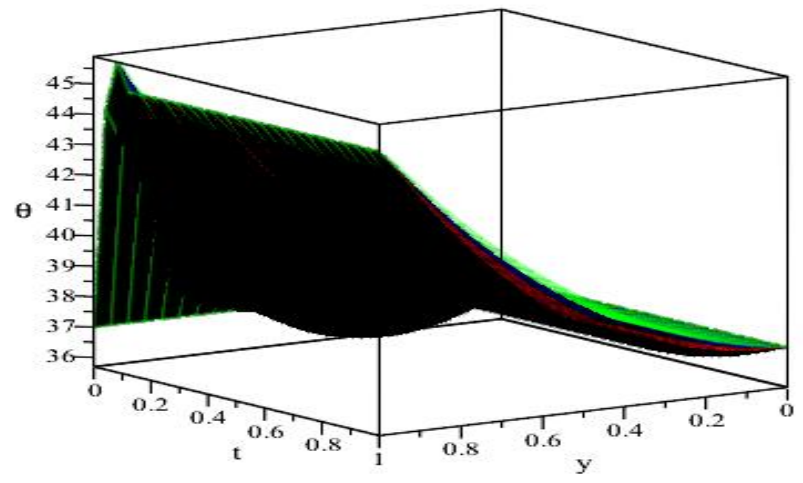

(a)

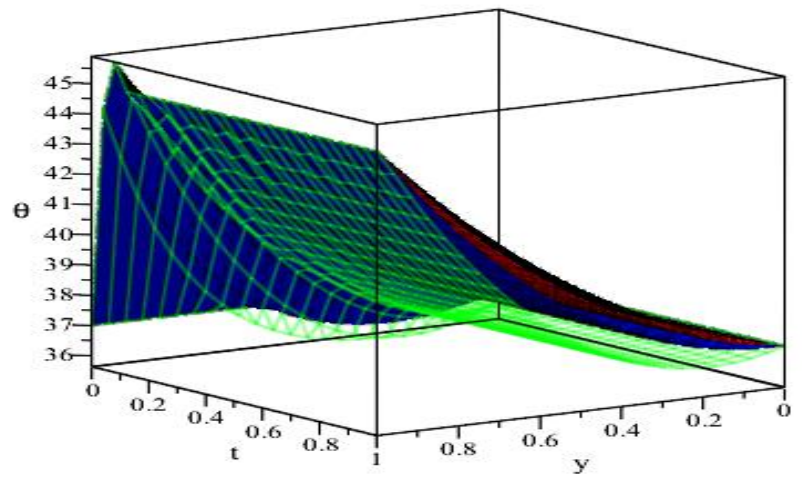

(b)

Figure 2: Transient and Steady state on Temperature profiles

\section{Nomenclature}

$\rho_{b}=$ Density of blood, $\left(\mathrm{Kgm}^{-3}\right)$

$C_{p b}=$ Blood specific heat at a constant pressure, $\left(J K g^{-1}\left({ }^{o} C\right)^{-1}\right)$

$K_{b}=$ Blood thermal conductivity, $\left(W m^{-1} K^{-1}\right)$

$\omega_{b}=$ Blood volumetric perfusion rate $\left(K_{g s}{ }^{-1}\right)$

$T_{b}=$ Arterial blood temperature, $\left({ }^{\circ} \mathrm{C}\right)$

$T=$ Local tissue temperature, $\left({ }^{\circ} \mathrm{C}\right)$

$k=$ Permeability of the porous medium

$\epsilon=$ Porosity of the tissue 


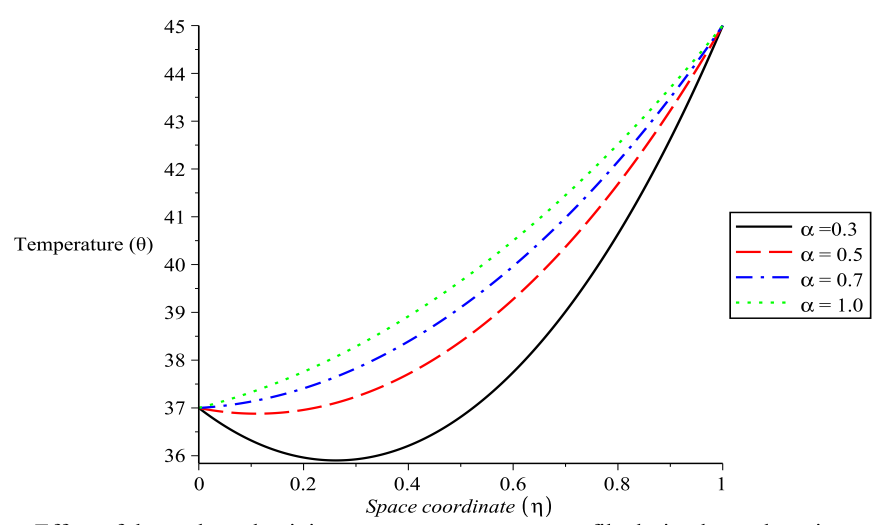

Effect of thermal conductivity terms on temperature profile during hyperthermia treatment of tumor at $\lambda=0.5$

(a)

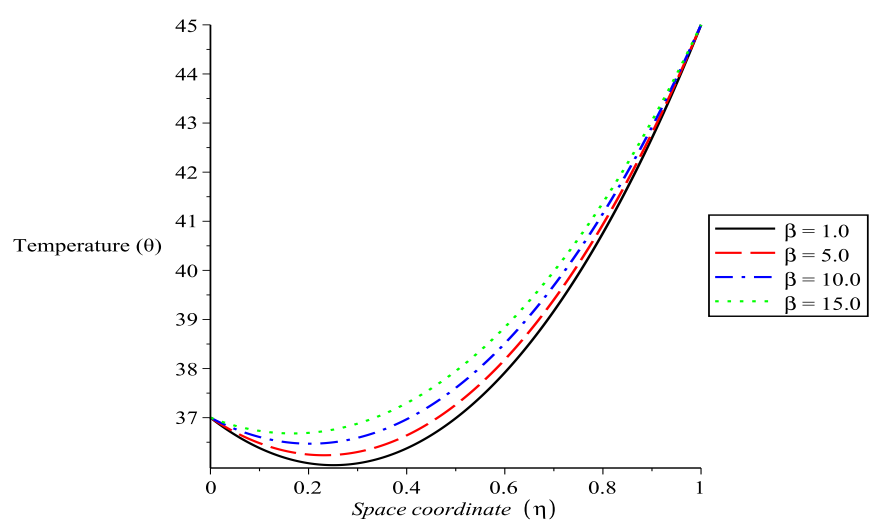

Effect of porosity terms on temperature profile during hyperthermia treatment of tumor at $\lambda=0.5$

(c)

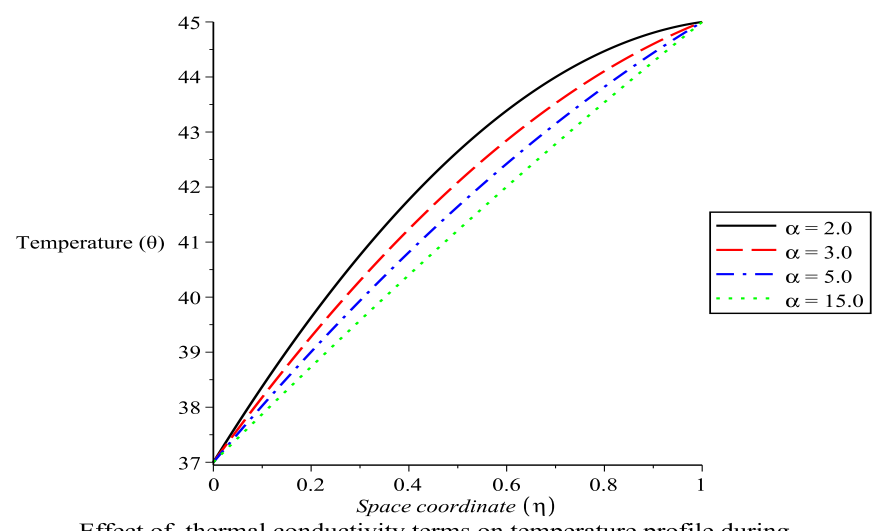

Effect of thermal conductivity terms on temperature profile during

hyperthermia treatment of tumor at $\lambda=2$

(b)

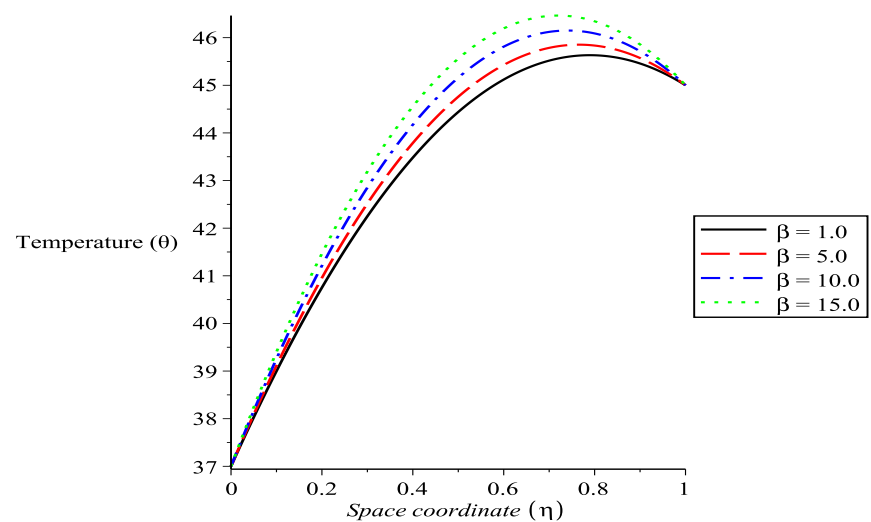

Effect of porosity terms on temperature profile during hyperthermia treatment of tumor at $\lambda=2$

(d)

Figure 3: The effects of Blood thermal conductivity and Porosity on Temperature profiles 


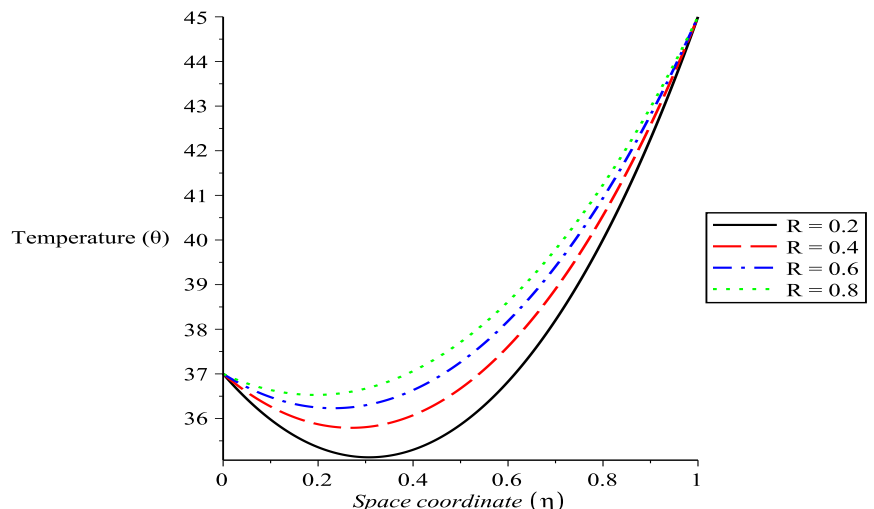

Effect of radiation parameters on temperature profile during hyperthermia treatment of tumor at $\lambda=0.5$

(a)

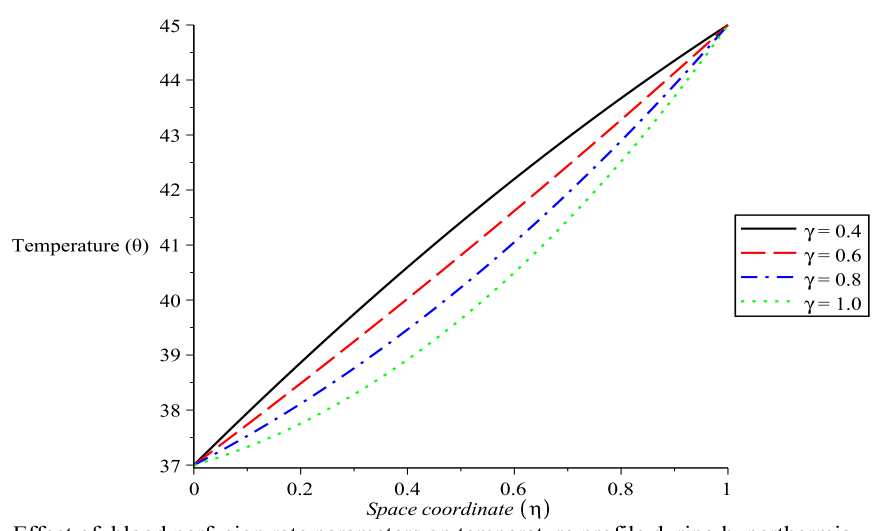

Effect of blood perfusion rate parameters on temperature profile during hyperthermia treatment of tumor at $\lambda=0.5$

(c)

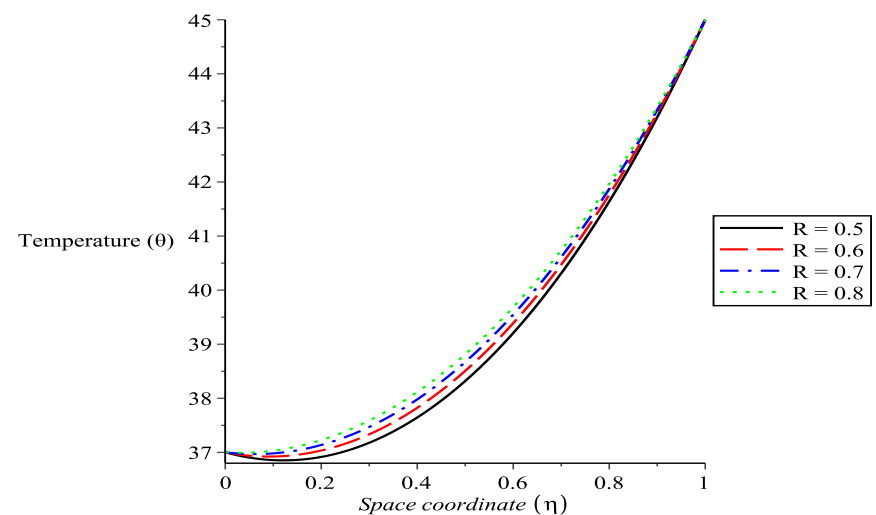

Effect of radiation parameters on temperature profile during hyperthermia treatment of tumor at $\lambda=1$

(b)

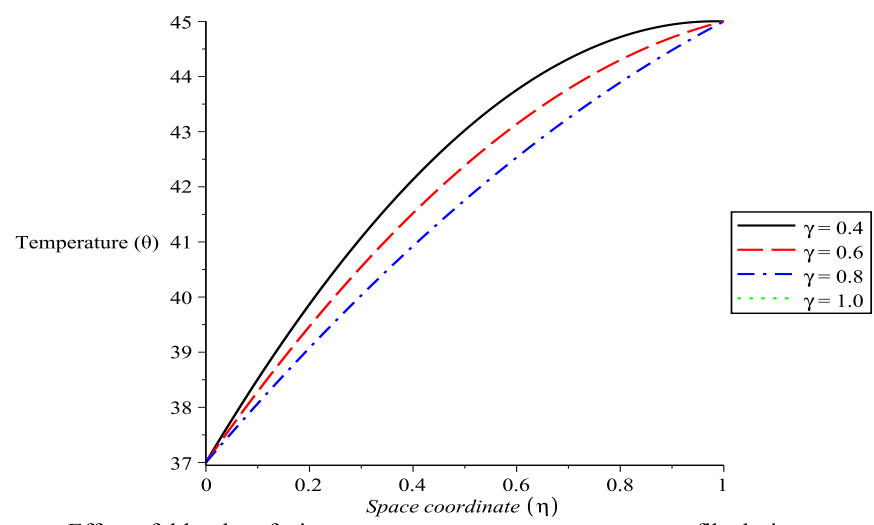

Effect of blood perfusion rate parameters on temperature profile during hyperthermia treatment of tumor at $\lambda=1$

(d)

Figure 4: The effects of Radiation parameters and Blood perfusion on Temperature profiles

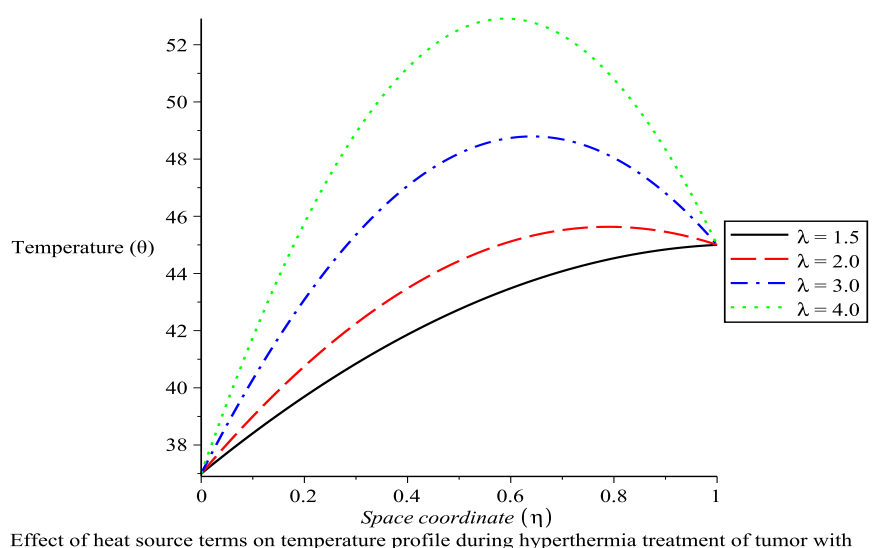

Space coordinate $(\eta)$
Effect of heat source terms on temperature profile during hyp
defarameter values

(a)

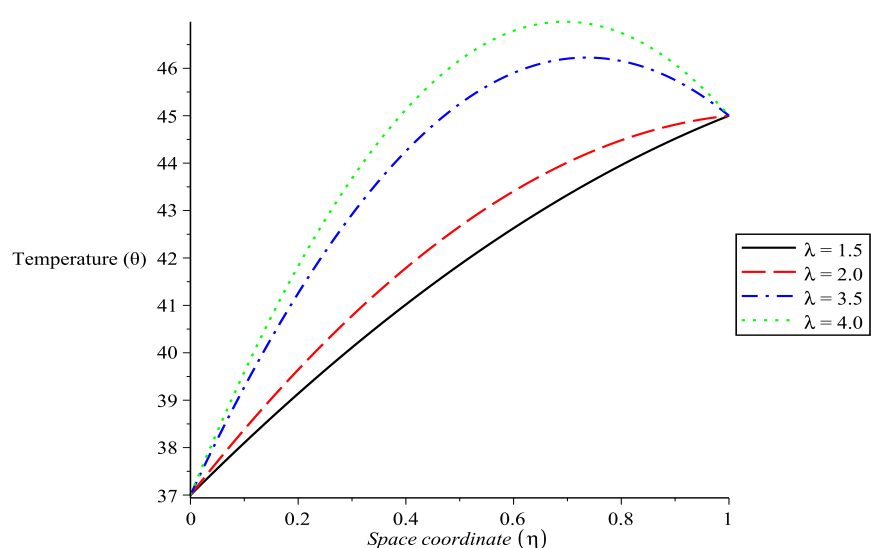

Effect of heat source terms on temperature profile during hyperthermia treatment of tumor at $\alpha=2$

(b)

Figure 5: The effects of Heat source on Temperature profiles 
$T_{o}=$ Reference temperature, $\left(T_{b}>T_{o}\right)\left({ }^{o} C\right)$

$Q_{m}=$ Heat source due to metabolic heat generation in the tissue, $\left(\mathrm{Wm}^{-3}\right)$

$q=$ Radiative heat flux, $\left(W m^{-2}\right)$

$R=$ Radiation parameters

$y=$ space coordinate,$(m)$

$t=$ time, $(s)$

$\alpha=$ Blood thermal conductivity terms

$\beta=$ Porosity parameters

$\gamma=$ Blood perfusion rate terms

$\lambda=$ Heat source terms 


\section{References}

[1] World Health Organisation ( WHO cancer fact \& figures,2017)

[2] Oke, S.I., Matadi, M.B. and Xulu, S.S., 2018. Optimal Control Analysis of a Mathematical Model for Breast Cancer. Mathematical and Computational Applications, 23(2), p.21.

[3] National Breast Cancer Foundation (NBCF Report, 2010)

[4] National Breast Cancer Foundation (NBCF Report, 2016)

[5] American Cancer Society.Cancer Facts and Figures 2014 - 2015. Atlanta, Georgia, 2016

[6] American Cancer Society. Breast Cancer Facts and Figures 2008 ? 2009. Atlanta Georgia,2010

[7] Xu F, Lu TJ, Seffen KA Biothermomechanics of skin tissues J.Mech Phys.Solids 2008a. $56,1852-1884$.

[8] Kumar C.S.S.R; Mohammad F. Magnetic nanomaterials for hyperthermia based therapy and controlled drug delivery. 2001. Adv. Drug Deliv. Rev. 63, 789-808

[9] Habash RWY, Bansal R, Krewaski O, Alhafid HT. Thermal therapy part I: an introduction of thermal thermal. Crit Rev Biomed Eng 2006a 34 (6): 459-489

[10] Habash RWY,Bansal R,Krewaski D, Alhafid HT. Thermal therapy part II : hyperthermia techniques.Crit Rev Biomed Eng 2006b, 34(6) : 491-542.

[11] Bhownik A, Singh R, Repaka R, Mishra SC. Conventional and newly developed bioheat transport models in vascularized tissues: a review.J. Therm.Biol. 2013. 38, 107125.

[12] Pennes HH Analysis of tissue and arterial blood temperatures in the resting human forearm 1948. J.Appl Physiol 85 : 5-34.

[13] Cattaneo C Surune forme de l'equation de la chaleur elinant le paradoxe d'une propagation instatance 1958 C.R Acad Sci 247: 431-432

[14] Vernotte P Les paradoxes de la theorie continue de l'equation de la chaleur 1958. C.R.Acad Sci 246 : 3145-3155.

[15] Tzou DY Macro-to Microscale heat transfer, 1996, Vol 50. Taylor \& Francis, Washington, DC.

[16] Adegbile E.A , Ayeni R.O, and Aregbesola Y.A.S. Steady state Temperature Rises for model biological tissues under Microwaves Hyperthermic Treatment. Journal of Science Forum 2004.

[17] Hill,James M., and Pincombe A.H. Some similarity temperature profiles for the microwave heating of a half-space. J.Austral Math. Soc. Ser. B33, 1992, 290-320.

[18] Strohbehn,J.W,Trembly, B.S, and Douple B.B. Blood flow effects on the temperature distribution from an invasive microwave antenna array used in cancer therapy IEEE Trans Biomed Eng. 1982, 29,no 9

[19] Foster K.R, Kritikos H.N, and Schwan H.P. Effect of surface cooling and blood flow on the microwave heating of tissue. IEEE Trans.Biomed Eng. 1978, 25, No 3, 313-316. 
[20] Kritikos H.N, Foster K.R, and Schwan H.P. Temperature profiles in spheres due to electromagnetic heating. JL.Z Microwave power 1981, 16, No 3 \& 4, 327-340

[21] Marchant T, Liu B. On the heating of two- dimensional slab in a microwave cavity : aprature effects. Anziam J.2002,43:137-148 .

[22] El-dabe Nabil T.M , Mohammed Mona A.A and El-Sayed Asma F. Effects of microwave heating on the thermal states of biological tissues. African Journal of Biotechnology, 2003, Vol 2(11), pp453-459.

[23] Adegbile E.A \& Ogunmoyela J.K. Thermoregulation in biological tissues during microwave hyperthermia, part I. Spatial dependent blood perfusion effect, Abacus 2005a

[24] Popoola A.O , Ayeni O.B ,?A note on the Multiplicity of Solutions of a Boundary Value Problem Arising From the Theory of Microwave Heating of Cancerous Tumor?. The International Institute for Science, Technology and Education Journals 2013; vol 3,No 14 pages $113-116$

[25] Makinde, O.D. and Animasaun, I.L., 2016. Bioconvection in MHD nanofluid flow with nonlinear thermal radiation and quartic autocatalysis chemical reaction past an upper surface of a paraboloid of revolution. International Journal of Thermal Sciences, 109, pp.159-171.

[26] A.R.A.Khaled \& K. Vafai . The role of porous media in modeling flow \& heat transfer in biological tissues. Int. j.Heat Mass Transfer. 2003, 46, 4989-5003.

[27] Animasaun, I.L., 2015. Effects of thermophoresis, variable viscosity and thermal conductivity on free convective heat and mass transfer of non-darcian MHD dissipative Casson fluid flow with suction and nth order of chemical reaction. Journal of the Nigerian Mathematical Society, 34(1), pp.11-31.

[28] Salawu, S.O. and Oke, S.I., 2018. Inherent Irreversibility of Exothermic Chemical Reactive Third-Grade Poiseuille Flow of a Variable Viscosity with Convective Cooling. Journal of Applied and Computational Mechanics, 4(3), pp.167-174.

[29] Salawu SO \& Dada MS,Radiative heat transfer of variable viscosity and thermal conductivity effects on inclined magnetic field with dissipation in a non-Darcy medium 2015, JNMS,1-14

[30] Chinyoka, T., Renardy, Y.Y., Renardy, M. and Khismatullin, D.B., 2005. Twodimensional study of drop deformation under simple shear for Oldroyd-B liquids. Journal of Non-Newtonian Fluid Mechanics, 130(1), pp.45-56.

[31] Chinyoka, T., 2008. Computational dynamics of a thermally decomposable viscoelastic lubricant under shear. Journal of Fluids Engineering, 130(12), p.121201.

[32] Gupta PK, Singh J, Rai KN. A numerical study on heat transfer in tissues during hyperthermia 2013. J Math Comput Model 57 : 1018-1037

[33] Ping Yuan. Numerical analysis of an equivalent heat transfer coefficient in a porous model for simulating a biological tissue in a hyper themia therapy 2009. Int.J Heat Mass Transfer 52.1734-1740. 